Nadeln erstarrende Rückstand betrug nach dem Trocknen auf Ton und mehrmaligem Umkristallisieren aus Wasser $65 \mathrm{mg}$.

Eine höhere Ausbeute wurde durch Zusatz von Maiseiweiß zu dem Nährboden erzielt. Dabei gingen wir folgendermaßen vor: Eine Anzahl sog. PenicillinKolben (Schott \& Gen., Jena) wurde mit je $1,5 l$ Substrat beschickt, und zwar: $4 \%$ Glucose, $0,2 \%$ $\mathrm{NaNO}_{3}, 0,1 \% \mathrm{KH}_{2} \mathrm{PO}_{4}, 0,05 \% \quad \mathrm{MgSO}_{4} \cdot 7 \mathrm{H}_{2} \mathrm{O}, 0,05 \%$ $\mathrm{KCl}, 0,001 \% \quad \mathrm{FeSO}_{4} \cdot 7 \mathrm{H}_{2} \mathrm{O}, 0,001 \% \mathrm{ZnSO}_{4} \cdot 7 \mathrm{H}_{2} \mathrm{O}$ und $0,25 \%$ Maiseiweiß ${ }^{5}$. Nach dem Sterilisieren wurde mit einer Sporensuspension aus einer 5 Tage alten Bierwürze-Agar-Kultur beimpft. Nach 10-tägigem Verweilen bei $25^{\circ}$ erhielten wir aus 2,5 $l$ Kulturfiltrat (= $100 \mathrm{~g}$ Glucose) in gleicher Weise (wie oben beschrieben) 3,4 g Rohprodukt. Nach mehrmaligem Umkristallisieren aus Wasser wurden farblose, lange Nadeln erhalten. Schmp. $170-171^{\circ}$; gut löslich in Äther, Äthanol und Äthylacetat. Die wäßrige Lösung der Substanz gab mit Bromwasser einen Niederschlag und mit Eisenchlorid eine tief-violette Färbung.

5 Von den Maizena-Werken, Hamburg.
$\mathrm{C}_{8} \mathrm{H}_{8} \mathrm{O}_{3}$. Ber. Mol.-Gew. 152 ,

Gef. Mol.-Gew. 147,9 (nach Rast), 151,8 (durch Titration mit $n / 10$-Lauge).

Zur eindeutigen Identifizierung der Substanz als 6-Methyl-salicylsäure stellten wir nach Angaben von Anslow und Raistrick ${ }^{3}$ von der isolierten Substanz noch den Methyläther dar. Die Schmelzpunkte der 4 verschiedenen Methylsalicylsäuren liegen z.Tl. recht nahe beieinander: $169^{\circ}$ (6-Methyl-salicylsäure), $167^{\circ}, 153^{\circ}$ und $177^{\circ}$. Weitaus geeigneter zur Unterscheidung sind die Methyläther, deren Schmelzpunkte recht weit auseinander liegen: $139^{\circ}$ (6-Methyl-salicylsäure-methyläther), $85^{\circ}, 70^{\circ}$ und $104^{\circ}$. Der aus der von uns isolierten Substanz erhaltene Methyläther schmolz bei $140^{\circ}$. Dies spricht eindeutig für den Methyläther der 6-Methyl-salicylsäure. Außerdem stellten wir auch noch das Acetylderivat der jsolierten Säure her. Schmp. 129-130 ${ }^{\circ}$ (nach Literaturangabe $\left.131^{\circ}\right)$.

$$
\begin{array}{lll}
\mathrm{C}_{10} \mathrm{H}_{10} \mathrm{O}_{4} . & \text { Ber. C 61,85, } & \mathrm{H} \mathrm{5}, 15 . \\
& \text { Gef. C } 62,00,62,03, & \mathrm{H} \mathrm{5}, 18,5,30 .
\end{array}
$$

\title{
BERICHTE
}

\section{Über die Rolle von Kupfer und Mangan im Leben der höheren Pflanzen*}

$\mathrm{D}$ ie aus dem Biochemischen Institut Helsinki (Leiter Prof. Dr. A. I. Vi r t a n e n) hervorgegangene Arbeit behandelt im ersten Abschnitt das Vorkommen von Kupfer und Mangan in höheren Pflanzen. Der einleitenden Übersicht über frühere Untersuchungen sind umfangreiche Zahlenangaben über den $\mathrm{Cu}$ und Mn-Gehalt einiger Pflanzenteile aus diesen Arbeiten beigefügt. Da es nach der Meinung des Verf. nicht möglich sein wird, über die Rolle von Kupfer und Mangan im Stoffwechsel der höheren Pflanzen eine klare Vorstellung zu gewinnen, solange diese Elemente getrennt und nicht als eine physiologische Einheit zusammen mit dem ihnen nahe verwandten, wichtigen Bioelement Eisen betrachtet werden, wurde bei den eigenen Analysen das Eisen mit eingeschlossen. Diese wurden an 34 verschiedenen, sowohl wildwachsenden als auch kultivierten Pflanzen ausgeführt. Die Mehrzahl der analysierten Pflanzen wurde auf zwei verhältnismäßig engen Gebieten (Waldboden- und Gartenbodenbezirk von je etwa $100 \mathrm{~m}^{2}$ ) gesammelt. Als Bezugssysteme wurden das Trockengewicht, der Aschengehalt und der Stickstoff gehalt benutzt, ferner wurde der Kupfer- und Mangangehalt mit dem Eisengehalt des Materials verglichen (Tab. 1). Kupfer- und Mangan- sowie $p_{\mathrm{H}}$-Bestimmungen von den betreffenden Bodenproben wurden ebenfalls ausgeführt (Tab. 2). Die Grenzwerte in allen Analysen (bezogen auf das

* Bericht über die gleichnamige Arbeit von J. Erkam a in Suomalaisen Tiedeakatemian Toimituksia, Sarja A, II. Chemica, 25, 1-105 [1947].
Trockengewicht) liegen für Mangan um das 440-fache, für Eisen um das 40-fache und für Kupfer nur um das 6-fache auseinander; das Kupfer weist also eine enge und Mangan eine sehr weite Schwankungsbreite auf (Tab.3). Daß auch hier der Standort auf den MnGehalt der Pflanzen Einfluß hat, ist aus Tab. 4 ersichtlich. Die auf neutralem Boden gewachsenen Pflanzen haben einen sehr niedrigen Mn-Gehalt, der aber, wie Tab. 2 erkennen läßt, nicht durch den MnMangel im Boden bedingt ist. Hinsichtlich der Stärke der Speicherung bestehen zwischen den einzelnen Arten und vielleicht auch zwischen höheren systematischen Gruppen Unterschiede, welche jedoch durch die Wirkung anderer Faktoren leicht überdeckt werden. Von den wildwachsenden Pflanzen waren in erster Linie Melampyrum silvaticum und auch Sedum telephium, Taraxacum officinale, Rumex acetosa und Convallaria majalis. von den Gartenpflanzen Gurke, Kürbis, Kartoffel, Karotte und Rhabarber als CuSpeicherpflanzen bemerkenswert. Hinsichtlich der MnSpeicherung waren die Unterschiede deutlicher, aber durch äußere Faktoren mehr überdeckt. Von den wildwachsenden Pflanzen enthielten Melampyrum silv. und Vaccinium-Arten am meisten Mangan; manganreich waren auch Oxalis acetosella und Rubus-Arten. Ein gewisser Antagonismus zwischen Kupfer und Mangan läßt sich annehmen, denn manche ziemlich viel Kupfer enthaltende Pflanzen (Sedum, Taraxacum, Rumex, Convallaria) waren arm an Mangan. Auch bestehen deutliche Unterschiede im $\mathrm{Cu}$ - und Mn-Gehalt ver- 


\begin{tabular}{|c|c|c|c|c|c|c|}
\hline \multirow[t]{2}{*}{ Name und Organ } & \multirow{2}{*}{$\begin{array}{c}p_{\mathrm{H}} \\
\text { des } \\
\text { Bodens }\end{array}$} & \multicolumn{3}{|c|}{$\begin{array}{c}\mathrm{mg} \text { in } 1 \mathrm{~kg} \\
\text { Trockensubstanz }\end{array}$} & \multirow[t]{2}{*}{$\mathrm{Cu}: \mathrm{Fe}$} & \multirow[t]{2}{*}{$\mathrm{Mn}: \mathrm{Fe}$} \\
\hline & & $\mathrm{Cu}$ & Mn & $\mathrm{Fe}$ & & \\
\hline \multicolumn{7}{|l|}{$\begin{array}{l}\text { a) Pflanzen von demselben Waldboden } \\
\qquad p_{\mathrm{H}} \mathbf{4 , 5}-\mathbf{5 , 4}\end{array}$} \\
\hline Convallaria majalis, Blatt ........... & 5,1 & 11,0 & 121 & 98 & 0,112 & 1,23 \\
\hline 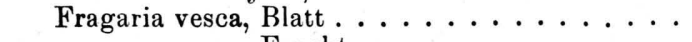 & 5,4 & 7,5 & 560 & 186 & 0,040 & 3,02 \\
\hline$, \quad, \quad$ Frucht $\ldots \ldots \ldots \ldots$ & 5,4 & 9,8 & 141 & 57 & 0,172 & 2,48 \\
\hline Linnaea borealis, Blatt. .......... & $4,5-5,2$ & 8,9 & 174 & 238 & 0,037 & 0,74 \\
\hline " $\quad " \quad$ Stengel $\ldots \ldots \ldots \ldots$ & $4,5-5,2$ & 12,5 & 380 & 164 & 0,076 & 2,32 \\
\hline Majanthemum bifolium, Blatt ........ & $4,5-5,2$ & 9,0 & 460 & 160 & 0,056 & 2,88 \\
\hline " $\quad$ "Stengel $\ldots \ldots \ldots$ & $4,5-5,2$ & $1(1,0$ & 182 & 71 & 0,141 & 2,56 \\
\hline Melampyrum silvaticum, Blatt $\ldots \ldots \ldots$ & $4,5-5,2$ & 31,7 & 1760 & 217 & 0,146 & 8,10 \\
\hline Orali" & $4,5-5,2$ & 18,0 & 673 & $\begin{array}{r}88 \\
122\end{array}$ & 0,202 & 7,65 \\
\hline $\begin{array}{l}\text { Oxalis acetosella, Blattspreite } \ldots \ldots \ldots \ldots \\
\text { Blattstiel } \ldots \ldots\end{array}$ & 5,1 & 6,7 & 628 & 133 & 0,051 & 4,72 \\
\hline 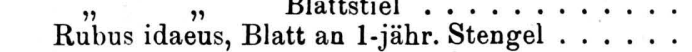 & $\begin{array}{l}5,1 \\
5,4\end{array}$ & $\begin{array}{l}6,8 \\
7,9\end{array}$ & $\begin{array}{l}202 \\
589\end{array}$ & $\begin{array}{r}67 \\
236\end{array}$ & $\begin{array}{l}0,102 \\
0,034\end{array}$ & $\begin{array}{l}3,76 \\
2,50\end{array}$ \\
\hline$" \quad " \quad$ Blatt an 2-jähr. Stengel . . . . . & 5,4 & 8,7 & 619 & 284 & 0,031 & 2,18 \\
\hline$" \quad, \quad$ Frucht $\ldots \ldots \ldots \ldots \ldots$ & 5,4 & 111,7 & 112 & 49 & 0,218 & 2,29 \\
\hline 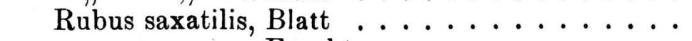 & 5,2 & 5,5 & 360 & 152 & 0,036 & 2,28 \\
\hline$" \quad " \quad$ Frucht $\ldots \ldots \ldots \ldots$ & 5,2 & 5,7 & 71 & 36 & 0,158 & 1,98 \\
\hline Rumex acetosa, Blattspreite $\ldots \ldots \ldots$ & 5,4 & 17,1 & 168 & 338 & 0,051 & 0,50 \\
\hline 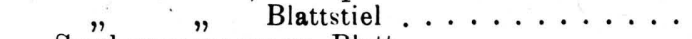 & 5,4 & 9,0 & 74 & 97 & 0,093 & 0,76 \\
\hline Sambucus racemosa, Blatt $\ldots \ldots \ldots$ & 5,4 & 8,7 & 374 & 262 & $(1,033$ & 1,43 \\
\hline 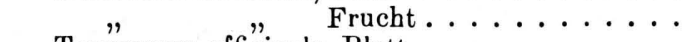 & 5,4 & 12,0 & 63 & 83 & 0,140 & 0,76 \\
\hline Taraxacum officinale, Blatt ......... & 5,4 & 17,6 & 79 & 254 & 0,069 & 0,31 \\
\hline . $\quad " \quad$ Wurzel ........ & 5,4 & 8,2 & 32 & 66 & 0,124 & 0,49 \\
\hline Trientalis europaea, Blatt $\ldots \ldots \ldots$ & $4,5-5,2$ & 10,9 & 298 & 165 & $0,0 \nmid 6$ & 1,81 \\
\hline ".. $\quad " . \quad$ Stengel ....... & $4,5-5,2$ & 1()$, 0$ & 150 & 72 & 0,139 & 2,08 \\
\hline Vaccinium myrtillus, Blatt $\ldots \ldots \ldots$ & $4.5-5,2$ & 10,2 & 740 & 106 & 0,096 & 7,00 \\
\hline$" \quad, \quad$ Stengel $\ldots \ldots \ldots$ & $4,5-5,2$ & 7,8 & 1184 & 93 & 0,086 & 12.74 \\
\hline 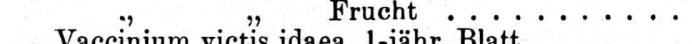 & $4,5-5,2$ & 4,2 & 138 & 18 & 0,234 & 7,56 \\
\hline Vaccinium victis idaea, 1-jähr. Blatt $\ldots \ldots$ & $4,5-5,2$ & 8,5 & 430 & 47 & 0,181 & 9,15 \\
\hline$" \quad 2$-jähr. Blatt $\cdots \cdots \cdot$ & $4,5-5,2$ & 5,7 & 1280 & 87 & 0,066 & 14,70 \\
\hline Stengel $\cdots \cdots \cdots \cdots$ & $4,5-5,2$ & 9,6 & 768 & $\begin{array}{r}107 \\
08\end{array}$ & $0,1,90$ & 7,20 \\
\hline$" \quad " \quad " \quad$ rrucen $\cdots \cdots \cdots \cdots$ & $4,0-0,2$ & $b, 7$ & 190 & 28 & 0,204 & 7,08 \\
\hline $\begin{array}{c}\text { b) Pflanzen von demselben Gartenboden } \\
\qquad p_{\mathrm{H}} 6,9-8,2\end{array}$ & & & & & & \\
\hline Allium schoenoprasum, Blatt . . . . . . . & 8,2 & 8,1 & 28 & 199 & 0,041 & 0,14 \\
\hline Cucumis sativus, Blatt $\ldots \ldots \ldots$ & 8,1 & 23,6 & 71 & 308 & 0,077 & 0,23 \\
\hline
\end{tabular}

Tab. 1. Analysen des

schiedener Pflanzenorgane. Die unterirdischen Organe waren gegenüber den Blättern arm an Kupfer und Mangan. Der $\mathrm{Cu}$ - und Mn-Gehalt der Blattstiele und Stengel waren im Vergleich zu den Blättern verschieden in Kräutern und in Holzgewächsen. Daß das Kupfer in der Frucht besonders in den Samen sich anhäuft, geht aus den mit Kürbis ausgeführten Analysen hervor. Bei mehrjährigen Blättern scheint der Mn-Gehalt in älteren Blättern zuzunehmen, der $\mathrm{Cu}$ Gehalt dagegen abzunehmen. Zwischen dem Wassergehalt und dem $\mathrm{Cu}$-Gehalt der Pflanzen besteht eine positive, zwischen dem Wassergehalt und Mn-Gehalt der Pflanzen eine negative Korrelation. Weiter wurde gefunden, daß zwischen dem Mn-Gehalt der Asche und dem Aschengehalt der Blätter eine sehr augenfällige negative Korrelation besteht und daß die Vorherrschaft des Mangans über das Eisen desto größer ist, je weniger Mineralstoffe das Blatt enthält (Tab. 5). Der auf Stickstoff berechnete Mn-Gehalt steht im um- gekehrten Verhältnis zu dem N-Gehalt, sowohl in Blättern als auch in Früchten; der auf Stickstoff bezogene $\mathrm{Cu}$-Gehalt ist dagegen ziemlich konstant, jedoch haben die stickstoffreichsten Blätter im allgemeinen auch den höchsten Cu-Gehalt. Die letzterwähnten Verhältnisse deuten darauf hin, daß das Kupfer in der Zelle wahrscheinlich gänzlich an Eiweiß, das Mangan hingegen wahrscheinlich größtenteils nicht organisch gebunden ist.

Im zweiten Abschnitt werden die Ergebnisse der Untersuchungen über den Einfluß von Kupfer und Mangan auf das Wachstum der Erbse in Wassegrkulturen mitgeteilt und diskutiert. In Einsalzlösungen ist Kupfer nachteilig in allen verwendeten Konzentrationen (bis $1: 10^{10}$ ). Mangan bewirkt in Konzentrationen von $1: 10^{7}$ und darüber Schädigungen in der Wurzel, dagegen wird die Sproßbildung und das Streckenwachstum erst bei Konzentrationen ab $1: 10^{6}$ erschwert. In Einsalzlösungen fördert Kupfer das 


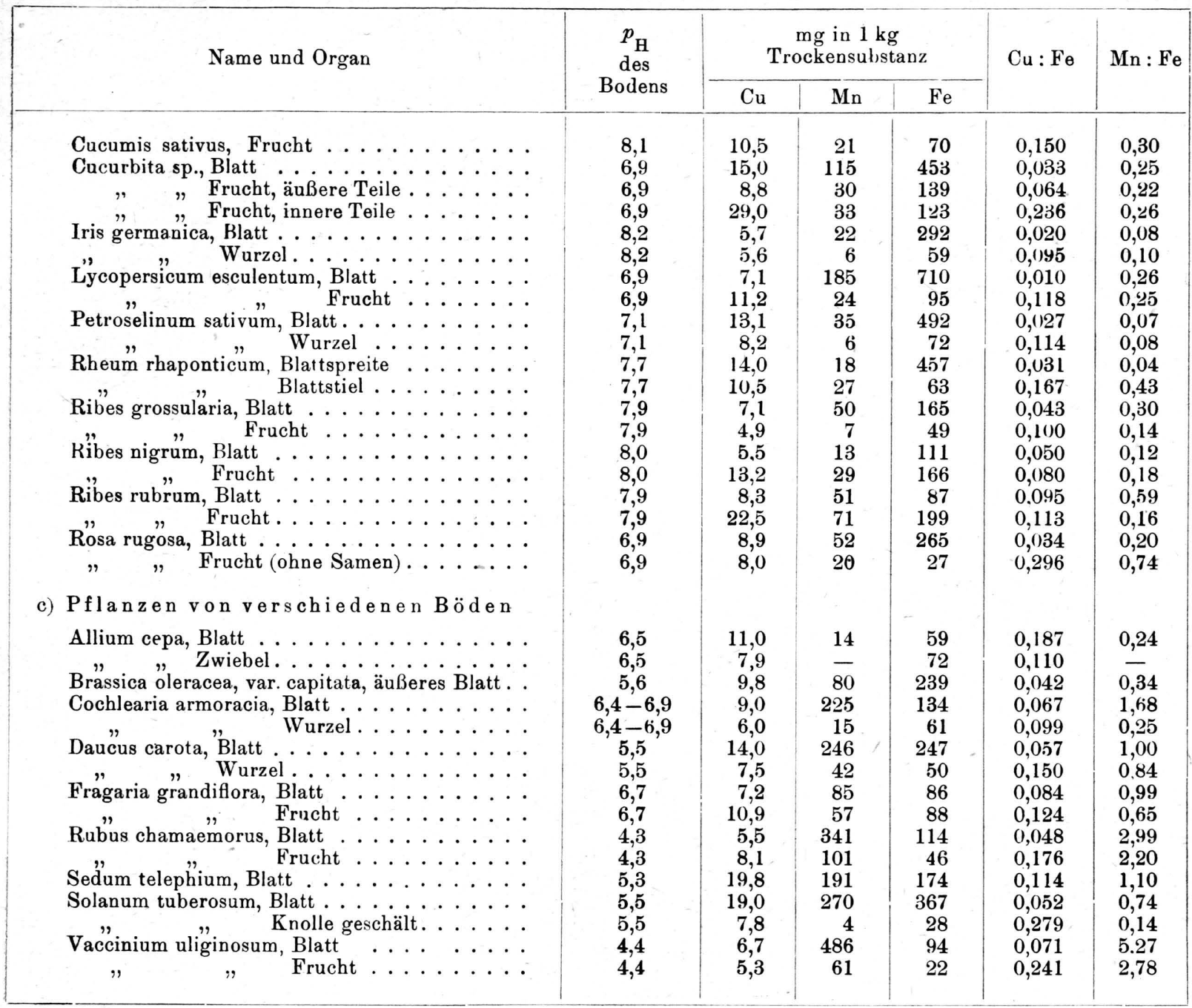

Pflanzenmaterials.

Wurzelwachstum, hemmt die Verbrennung der Samenreservestoffe und verhindert die Wasseraufnahme. In vollständiger Nährlösung werden die Giftwirkungen von Kupfer und Mangan weitgehend eliminiert. Sowohl Kupfer wie Mangan sind unbedingt notwendig für die normale Entwicklung der Erbse. Das Fehlen des Kupfers verursacht vor allem Sterilität der Pflanzen, bei $\mathrm{Cu}$-Mangel geht die Keimfähigkeit der Samen verloren. Die Cu-Düngung verlangsamt das Längenwachstum des Stengels, fördert jedoch das Gesamtwachstum und bringt einen erhöhten Wassergehalt sowie eine höhere Salzaufnahme mit sich. Der MnMangel hindert vor allem das vegetative Wachstum, fördert die Trockensubstanzbildung und vermindert den Aschengehalt der Pflanze. Das Kupfer fördert in der Pflanze die Eisenaufnahme, hemmt aber die Manganaufnahme. Mangan in der Pflanze hemmt sowohl Kupfer- als auch Eisenaufnahme. Das vermehrt aufgenommene Mangan verjagt $\mathrm{z}$. Tl. das Kupfer und
Eisen aus den Blättern. Das Mangan selbst scheint nicht leicht von Blatt zu Blatt zu wandern, sondern bleibt in den absterbenden älteren Blättern zurück. Bei dem Stoffwechsel der Pflanze stehen das Kupfer und das Mangan in einem weitgehenden Abhängigkeitsverhältnis zum Eisen, welches Element mit den zwei erstgenannten in der lebenden Pflanzenzelle eine vielleicht ebenso charakteristische Einheit bildet wie die drei lebenswichtigen Antagonisten Kalium, Calcium und Magnesium. Die Krankheitssymptome, vor allem die verschiedenen Formen der Chlorose, die auf $\mathrm{Cu}$ - bzw. Mn-Mangel oder $\mathrm{Cu}$ - bzw. Mn-Überschuß beruhen, kënnen letzten Endes auf eine Störung des Gleichgewichtes im Ferro-Ferri-Redoxsystem des Protoplasmas bzw. des Zellsaftes zurückgeführt werden, wofür das umseitige Schema entworfen wird (S. 384 oben).

Der dritte und letzte Abschnitt befaßt sich mit der Einwirkung von Kupfer und Mangan auf die Bildung 


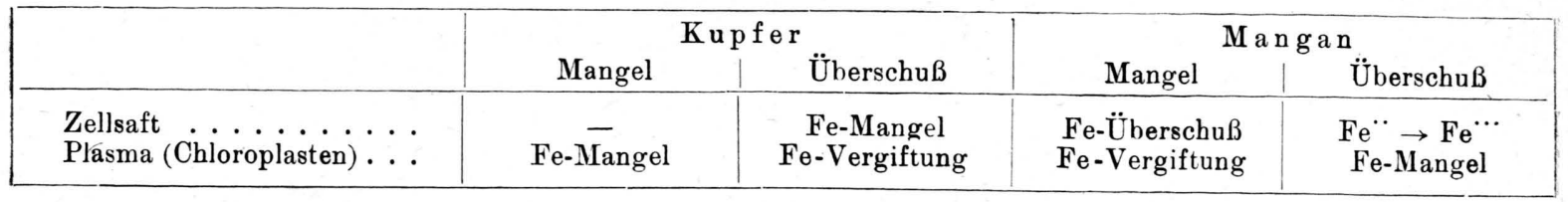

einiger Biokatalysatoren im Stoffwechsel der höheren Pflanze. Verf. gibt zunächst einen Überblick über die bekannten Enzyme, in welchen Kupfer oder Mangan als aktive Gruppe bzw. als Aktivator eine Rolle spielen. Demnach gilt Kupfer als Aktivator der Laccase, Tyrosinase und Brenzcatechinoxydase, Ascorbinsäureoxydase sowie Cytochromoxydase, Mangan als Aktivator von Arginase, Dipeptidasen, Phosphatasen, von Enolase, Carboxylase, Isocitricodehydrase, Phosphogluco-mutase sowie von Dioxymaleinsäureoxydase. Das Kupfer scheint immer fest an die Eiweißkomponente des Ferments gebunden zu sein, so daß es als prosthetische Gruppe und Wirkungsgruppe aufzufassen ist. Dagegen ist das Mangan, das immer als zweiwertiges Ion wirkt, locker gebunden und nur als ein zur optimalen Wirkung des Fermentes unerläßliches Komplement anzusehen. In den meisten Fällen ist es möglich, das Mangan durch ein anderes zweiwertiges Metall, wie Magnesium, Eisen oder Kobalt, zu ersetzen. Die eigenen Untersuchungen beschäftigten sich mit den Einwirkungen von Kupfer und Mangan auf die Bildung und den Zerfall der Ascorbinsäure, auf die katalytische und peroxydatische Aktivität der Pflanzengewebe sowie auf den Pigmentgehalt der Blätter. Zwischen dem Ascorbinsäuregehalt bzw. der Katalaseoder Peroxydaseaktivität und dem Kupfer- und Mangangehalt verschiedener Pflanzen und Pflanzenteile sind keine Korrelationen festzustellen. Bei der Erbse in Wasserkultur fördert das Mangan deutlich die

\begin{tabular}{|c|c|c|c|c|c|c|c|}
\hline \multicolumn{4}{|c|}{ Waldboden $\left(p_{\mathrm{H}} 4,5-5,4\right)$} & \multicolumn{4}{|c|}{ Gartenboden $\left(p_{\mathrm{H}}, 6,9-8,2\right)$} \\
\hline \multirow{2}{*}{$\begin{array}{l}\text { Boden- } \\
\text { probe } \\
\text { Nr. }\end{array}$} & \multicolumn{3}{|c|}{$\begin{array}{c}\text { in } 1 \mathrm{~kg} \text { Trocken- } \\
\text { substanz }\end{array}$} & \multirow{2}{*}{$\begin{array}{c}\text { Boden- } \\
\text { probe } \\
\text { Nr. }\end{array}$} & \multicolumn{3}{|c|}{$\begin{array}{c}\text { in } 1 \text { kg Trocken- } \\
\text { substanz }\end{array}$} \\
\hline & $\mathrm{mg} \mathrm{Cu}$ & $\mathrm{mg}_{\beta} \mathrm{Mn}$ & & & $\mathrm{mg} \mathrm{Cu}$ & mg Mn & \\
\hline $\begin{array}{l}1 \\
2 \\
3\end{array}$ & & $\begin{array}{l}0<4 \\
597 \\
650\end{array}$ & & $\begin{array}{l}1 \\
2 \\
3\end{array}$ & & $\begin{array}{l}624 \\
816 \\
634\end{array}$ & 9,20 \\
\hline Mittel & 24,5 & 623,7 & 1,89 & Mittel & & 691,6 & 9,20 \\
\hline
\end{tabular}

Tab. 2. Bodenanalysen.

\begin{tabular}{|c|c|c|c|c|c|c|}
\hline & \multirow{2}{*}{ 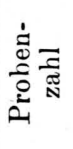 } & \multicolumn{3}{|c|}{$\begin{array}{c}\mathrm{mg} \text { in } 1 \mathrm{~kg} \text { Trocken- } \\
\text { substanz }\end{array}$} & \multicolumn{2}{|c|}{$\begin{array}{l}\text { Durchschnittl. } \\
\text { Abweichung }\end{array}$} \\
\hline & & $\begin{array}{c}\text { Mittel- } \\
\text { wert }\end{array}$ & Max. & Min. & $\mathrm{mg}$ & $\%$ \\
\hline & 69 & 10,5 & 31 & 5,3 & $\pm \quad 3,8$ & \pm 36 \\
\hline Mangan & 68 & 237,5 & 1760,0 & 4,0 & $\pm 235,6$ & \pm 95 \\
\hline
\end{tabular}

Tab. 3. Schwankungen im Cu- und Mn-Gehalt der Pflanzenproben.
Ascorbinsäurebildung, die bei Mn-Mangel wesentlich herabgesetzt ist. Die Kupferdüngung dagegen scheint keinen Einfluß auf die Bildung der Ascorbinsäure zu haben. Sowohl die Kupfer- als auch die Mangandüngung, vor allem die erstere, vermindern das Ascorbinsäure-Oxydationsvermögen der Erbsengewebe. Dieser Einfluß ist auf eine Hemmung der peroxydatischen Oxydationen zurückzuführen, die hinsichtlich des Kupfers schon in der Keimungsphase, hinsichtlich des Mangans erst bei älteren Pflanzen warzunehmen ist. Der Ascorbinsäure-Oxydationsmechanismus kann sich bei der Erbse vollständig durch die Annahme einer peroxydatischen Oxydation erklären, ohne daß man die Wirkung einer spezifischen Oxydase oder einer Metallkatalyse mit in Rechnung $\mathrm{zu}$ ziehen braucht. Auf die Katalaseaktivität der Erbsenkeimlinge haben weder Kupfer noch Mangan in vollständiger Nährlösung einen nennenswerten Einfluß. Bei ausgewachsenen Erbsen ist die Katalaseaktivität der grünen $\mathrm{Cu}$ - bzw. Mn-Mangelblätter höher als die der vollständig gedüngten. Bei $\mathrm{Cu}$ - bzw. Mn-Mangel sinkt in den Erbsenblättern der Gehalt an Chlorophyll a und b ziemlich gleichmäßig. In derselben Weise ver-

\begin{tabular}{|c|c|c|c|c|c|}
\hline \multirow[t]{2}{*}{$f$} & \multirow[t]{2}{*}{ Standort } & \multirow{2}{*}{ 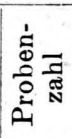 } & \multicolumn{3}{|c|}{$\begin{array}{l}\mathrm{mg} \text { in } 1 \mathrm{~kg} \\
\text { Trockensubstanz }\end{array}$} \\
\hline & & & $\mathrm{Cu}$ & Mn & $\mathrm{Fe}$ \\
\hline \multirow[t]{2}{*}{ Blätter } & $\left\{\begin{array}{l}\text { Waldboden } \\
p_{\mathrm{H}} 4,5-5,4\end{array}\right.$ & 16 & 11,0 & 540 & 185 \\
\hline & & 11 & 10,6 & 58 & 322 \\
\hline \multirow{2}{*}{ Früchte } & $\left\{\begin{array}{l}\text { Waldboden } \\
p_{\mathrm{H}} 4, \mathbf{5}-\mathbf{5 , 4}\end{array}\right.$ & 6 & 8,0 & 121 & 45 \\
\hline & $\left(\begin{array}{l}\text { Gartenboden } \\
p_{\mathrm{H}}\end{array}\right.$ & 8 & 13,5 & 24 & 109 \\
\hline
\end{tabular}

Tab.4. Einfluß des Standortes auf den Cu-, Mn- und Fe-Gehalt der Pflanzen.

\begin{tabular}{|c|c|c|c|c|c|}
\hline \multirow{2}{*}{\multicolumn{2}{|c|}{ Standort }} & \multirow{2}{*}{ 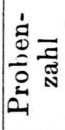 } & \multirow{2}{*}{$\begin{array}{c}\text { Mittlerer } \\
\text { Aschen- } \\
\text { gehalt } \\
\%\end{array}$} & \multicolumn{2}{|c|}{$\begin{array}{l}\text { In } 100 \mathrm{~g} \\
\text { Rohasche }\end{array}$} \\
\hline & & & & Cu mg & Mn mg \\
\hline Blätter & $\begin{array}{l}\text { Waldboden } \\
\text { Gartenboden }\end{array}$ & $\begin{array}{l}16 \\
11\end{array}$ & $\begin{array}{r}8,77 \\
20,12\end{array}$ & $\begin{array}{r}13,6 \\
6,7\end{array}$ & $\begin{array}{r}873 \\
33\end{array}$ \\
\hline Früchte & $\begin{array}{l}\text { Waldboden } \\
\text { Gartenboden }\end{array}$ & $\begin{array}{l}6 \\
8\end{array}$ & $\begin{array}{r}4,02 \\
12,14\end{array}$ & $\begin{array}{l}22,2 \\
11,6\end{array}$ & $\begin{array}{r}329 \\
22\end{array}$ \\
\hline
\end{tabular}

Tab.5. Mittlerer Aschengehalt der Pflanzenteile und $\mathrm{Cu}$ - bzw. Mn-Gehalt der Asche. 
halten sich auch die gelben Blattpigmente bei dem Mn-Mangel. Die gelben Pigmente scheinen empfindlicher gegen den Mn-Mangel zu sein als die grünen. Bei Cu-Mangel hat dagegen von den gelben Pigmenten nur das Carotin abgenommen, das Xanthophyll scheint im Gegenteil zuzunehmen. Auf Grund seiner Untersuchungen gelangt Verf. zu folgendem Urteil: Die primären Wirkungen des Kupfers und Mangans im Lebensprozeß der Pflanze sind in der Eigenart ihrer Atome und Ionen zu suchen. Zusammen mit dem Eisen bilden sie die wichtigsten der in der Pflanze vorkommenden Übergangselemente. Die verhältnis- mäßig kleinen Ionen, die wechselnde Valenz, die große magnetische Suszeptibilität und das Komplexbildungsvermögen bilden den physikochemischen Grund ihrer biologischen Wirkungen. Eigenartig ist besonders ihre Neigung, vor allem bei komplexen Verbindungen freie Elektronen abzugeben oder zu empfangen und so als Elektronenvermittler zu wirken. Vielleicht noch wichtiger ist ihre Fähigkeit, eine diamagnetische Atomgruppe reversibel in einen äußerst aktiven radikalartigen Zustand mit starkem paramagnetischem Feld zu bringen.

Wilhelm Schropp, Weihenstephan.

\section{BESPRECHUNGEN}

Anorganische Strukturchemie. Von Walter H ü c k e l. Ferd. Enke Verlag, Stuttgart 1948. 1033 S. mit 170 Abb., Preis geh. DM 68.-,-geb. DM 71.20.

Mit dem vorliegenden Werk hat der Verf. verschiedener ausgezeichneter Lehrbücher sowie der bekannten „Theoretischen Grundlagen der organischen Chemie" eine Lücke geschlossen, welche seit langem in der deutschen chemischen Literatur bestanden hat. Es ist dies eine geschlossene Darstellung der großen Fortschritte und Erkenntnisse, welche auf dem Gebiet der anorganischen Struktur- und Konstitutionslehre bis in die neueste Zeit erzielt worden sind. Das Erscheinen dieses deutschen Werkes ist um so erfreulicher, als neuerdings auch die englische Fachliteratur ein Buch mit gleichlautendem Titel aufweist*.

In drei „Büchern“ und zwölf Kapiteln hat der Verf. ein außerordentlich umfangreiches Material eingeordnet. In die "Strukturchemie“ ist gleichzeitig das Problem der „Konstitution“ eingearbeitet, also der Inbegriff der räumlichen Lagerung der Atome bzw. Atomkerne und die Verteilung der sie bindenden Elektronen. Das erste Buch „Stöchiometrie und Systematik“ beginnt mit einem einleitenden Kapitel, in welchem die Grundprobleme der Chemie aus ihren historischen Wurzeln entwickelt werden. Nach dem 2. Kapitel, welches die Grundlage der Systematik aus valenztheoretischen Betrachtungen ableitet, folgt eine ausführliche Wiedergabe der klassischen Koordinationslehre Werners auf moderner Grundlage. Die wichtigsten Verbindungstypen ein- und mehrkerniger Komplexverbindungen sowie die Isomeriefälle bei anorganischen Molekülen werden erörtert. Das zweite Buch ist dem Atombau und der chemischen Bindung gewidmet. Im 4. Kapitel wird zunächst das Periodische System behandelt und die Struktur der Elektronenhülle der Atome in üblicher Weise aus spektralen Beobachtungen entwickelt. Der Kernbau, ebenso wie die neuen Transurane werden nur gestreift. Im 6. Kapitel gelangt der Verfasser - vielleicht etwas spät - zum Problem der Atombindung. Die Einfach-,

* A. F. Wells, Structural inorganic chemistry, Oxford, Clarendon Press 1945.
Doppelt- und Dreifach-Bindung werden mittels der quantenmechanischen Valenztheorie interpretiert, und das Auftreten von-Mesomerie bei anorganischen Molekülen wird aufgezeigt. Das vorangeschaltete 5. Kapitel gibt eine recht interessante Einführung in die physikalischen Methoden zur Erforschung der chemischen Bindung: Ermittlung der Raumerfüllung, Röntgenstrukturanalyse, Elektroneninterferometrie, Leitfähigkeitsund dielektrische Messungen, Lichtabsorption und -emission, Raman-Spektrum und magnetische Messungen. Das dritte Buch „Struktur und Konstitution“ beginnt im 7. Kapitel mit der Behandlung der leichtflüchtigen anorganischen Elemente und Verbindungen, wie insbesondere den flüchtigen Hydriden. Die Kapitel 8-10 umfassen den Stoff der Kristallchemie. Sie sollen zeigen, welche Bedeutung dieselbe im Rahmen der anorganischen Chemie besitzt. Das 8. Kapitel bringt die wichtigsten allgemeinen kristallchemischen Probleme. Es werden hier die Gittertypen binärer und ternärer Verbindungen, die Bedeutung der Ionenradien, die Begriffe der Morphotropie, Polymorphie und Isomorphie behandelt. Im 9. und 10. Kapitel folgen spezielle Gebiete, die Chemie der Silikate und Gläser, der Metalle und Legierungen und schließlich der nichtstöchiometrisch zusammengesetzten Oxyde, Sulfide, Nitride und Carbide. Im 11. Kapitel weist der Verf. auf die Zusammenhänge hin, welche bei anorganischen Stoffen zwischen Konstitution und Reaktivität bestehen. Es ist in Abschnitte über Gasreaktionen, Reaktionen in wäßrigen und nichtwäßrigen Lösungen und Umsetzungen mit festen Stoffen unterteilt. Im Schlußkapitel sind die strukturchemischen Denkweisen der organischen und der anorganischen Chemie einander gegenübergestellt. Eine große Zahl von Literaturhinweisen und ein sorgfältig ausgearbeitetes Register erhöhen den Wert des Buches.

Bei der Fülle des gegebenen Materials ist eine ins einzelne gehende Stellungnahme unmöglich. Den Studierenden wird vielleicht das Material etwas überwältigen, und für denjenigen, welcher mit manchem nicht von vorneherein vertraut ist, wird das Einlesen schwierig sein. Der Sachkenner jedoch wird das Werk mit großem Interesse und hohem Genuß lesen und aus der Reichhaltigkeit des Gebotenen viele Anregun- 